\title{
Halofuginone upregulates the expression of heparanase in thioacetamide-induced liver fibrosis in rats
}

\author{
Olga Ohayon ${ }^{1}$, Nidal Mawasi ${ }^{1,5}$, Anna Pevzner ${ }^{1}$, Ana Tryvitz ${ }^{1}$, Tsvia Gildor ${ }^{1}$, Mark Pines², Marcos Rojkind ${ }^{3,4}$, \\ Melia Paizi ${ }^{1}$ and Gadi Spira ${ }^{1}$
}

Advanced hepatic fibrosis is characterized by excessive extracellular matrix deposition, where collagen and proteoglycans are the main constituents of scar tissue. In previous studies, we showed that heparanase, a heparan sulfate-degrading enzyme, and vascular endothelial growth factor (VEGF) play an important role during liver development and remodeling. In this communication, we investigated the relationship between heparanase and VEGF in thioacetamide-induced liver fibrosis in rats. Our study shows that heparanase mRNA expression levels correlate with those of VEGF during the induction and recovery stages of liver fibrosis. We further demonstrated that treating fibrotic rat livers with halofuginone (HF), a multipotent antifibrogenic drug, and subsequently subjecting them to hydrodynamics-based transfection with human VEGF-165 resulted in elevated expression of heparanase mRNA. Moreover, these rats demonstrated an improved capacity to regenerate following $70 \%$ partial hepatectomy. In vitro, HF stimulated heparanase and VEGF mRNA expression in hepatic stellate cells. Taken together, our results suggest that in addition to the known multiple functions of HF, it also enhances heparanase and VEGF expression and promotes liver regeneration. Accordingly, HF seems to possess ideal properties required to become an excellent antifibrogenic agent in humans.

Laboratory Investigation (2008) 88, 627-633; doi:10.1038/labinvest.2008.30; published online 5 May 2008

KEYWORDS: halofuginone; heparanase; hepatic stellate cells; hydrodynamics-based transfection; rat liver fibrosis; VEGF

Hepatic fibrosis is a dynamic process characterized by excessive accumulation of interstitial extracellular matrix (ECM), distortion of normal hepatic architecture, hemodynamic alterations and hepatic dysfunction. In the late stages of the disease, collagen and proteoglycan content may be up to six times higher than that found in healthy livers. ${ }^{1}$ Other ECM components like non-fibril forming collagens, which include collagen type IV and laminin, also increase. ${ }^{2}$ Although most liver cells express various ECM components, hepatic stellate cells (HSCs) are the main producers of the ECM found in scar tissue of the liver. ${ }^{3}$

Chemically induced experimental liver fibrosis could be a reversible process when the pathogenic trigger is removed. ${ }^{4,5}$ While fibrotic neomatrix contains a wide range of collagenous and non-collagenous ECM proteins, remodeling of the recovering liver ECM components is largely attributed to decreases of collagen type I and tissue inhibitor of metalloproteinase (TIMP) 1 and $2 .^{6}$
Heparan sulfate proteoglycans constitute an important component of the ECM contributing to integrity and insolubility of ECM. Heparan sulfate proteoglycans consist of a core protein to which heparan sulfate side chains are covalently attached. In the ECM, heparan sulfate proteoglycans interact with various molecules, including fibronectin, laminin and collagen and serve as a storage depot of growth factors such as vascular endothelial growth factor (VEGF), bFGF, keratinocyte growth factor, hepatocyte growth factor (HGF) and transforming growth factor- $\beta$ (TGF- $\beta$ ). ${ }^{7,8} \mathrm{He}-$ paran sulfate moieties can be specifically cleaved off by heparanase, a mammalian endoglucuronidase, ${ }^{9,10}$ thereby controlling release of the bound growth factors and potentially affecting a multitude of biological ECM remodelingassociated processes.

Traditionally heparanase is correlated with the metastatic potential of tumor cells, ${ }^{9-12}$ although it was also found to facilitate cell invasion associated with autoimmunity, inflammation

\footnotetext{
${ }^{1}$ Department of Anatomy and Cell Biology, The Bruce Rappaport Faculty of Medicine, Technion-Israel Institute of Technology, Haifa, Israel; ${ }^{2}$ Institute of Animal Sciences, Agricultural Research Organization, the Volcani Center, Bet Dagan, Israel; ${ }^{3}$ Department of Biochemistry and Molecular Biology, George Washington University Medical Center, Washington, DC, USA and ${ }^{4}$ Department of Pathology, George Washington University Medical Center, Washington, DC, USA; ${ }^{5}$ Contributed equally to as first author.

Correspondence: Assoc. Professor G Spira, PhD, Department of Anatomy and Cell Biology, The Bruce Rappaport Faculty of Medicine, Technion-Israel Institute of Technology, PO Box 9649, Haifa 31096, Israel. E-mail: spira@tx.technion.ac.il
} 
and angiogenesis. ${ }^{10,13}$ The latter has been shown to be mediated by heparanase both through its enzymatic activity and by non-enzymatic pathways like Src activation, a known VEGF inducer. ${ }^{14}$ In a previous paper, we have documented a biphasic gain of heparanase expression following $70 \%$ partial hepatectomy $(\mathrm{PHx})$ associated with a corresponding increase in free heparan sulfate content. ${ }^{15}$

Halofuginone (HF) is an alkaloid originally isolated from the plant Dichroa febrifuga and routinely used as a coccidiostat in veterinary applications. As a specific inhibitor of collagen type I synthesis, HF has extensively been studied in the setting of experimental fibrosis. ${ }^{16-19}$ In the past we have demonstrated the beneficial effect of HF on fibrotic rat liver regeneration following $\mathrm{PHx} .{ }^{20}$

In the present study, we investigated whether the beneficial effects of HF on thioacetamide (TAA)-induced liver fibrosis stem from its already known antifibrogenic effects and its capacity to enhance regeneration via production of heparanase. We showed a direct correlation between the fluctuations of heparanase and VEGF during accumulation and resolution of scar tissue. We further showed that HF enhanced these changes. In addition, we demonstrated that liver cell transfection with VEGF in vivo upregulated heparanase expression and enhanced liver regeneration.

\section{MATERIALS AND METHODS}

HF bromhydrate was purchased from Collgard Biopharmaceuticals (Tel Aviv, Israel).

\section{Animal Experimentation}

Adult, male Sprague-Dawley rats (250-300g) were purchased from Harlan Laboratories (Jerusalem, Israel). Fibrosis was induced by intraperitoneal administration of TAA, $200 \mathrm{mg} / \mathrm{kg}$ body weight, twice weekly. Saline-injected animals served as control. HF, at $1 \mu \mathrm{g} / 100 \mathrm{~g}$ body weight, was injected intraperitoneally every 3-4 days. Saline-injected animals served as control. Seventy percent PHx was performed under light anesthesia by removing the median and left lateral lobes. The excised liver was weighed and samples were treated with $4 \%$ paraformaldehyde for histochemistry or frozen in liquid nitrogen for RNA extraction and hydroxyproline content. Animals were killed 5 days post hepatectomy and their livers were harvested for analysis. Sham operations did not have any effect on the outcome of liver regeneration in preliminary experiments, and thus were not routinely performed. Rats had free access to standard rat chow and tap water and received humane care. The protocol for animal experimentation was approved by the Ethical Committee of Technion-Israel Institute of Technology (permission number IL-063-06-2006).

\section{Grading Liver Fibrosis and Histology}

The Ishak scoring system was used to determine liver tissue damage, grading between 0 (normal histology) to 6 (Cirrhosis). ${ }^{21}$ Histological evaluation was performed on liver sections fixed in $4 \%$ formaldehyde/PBS, dehydrated in graded ethanol solutions, and following staining with Sirius Red. ${ }^{22}$

\section{Hydroxyproline}

Hydroxyproline was measured using a colorimetric assay as previously described. ${ }^{23,24}$ Briefly, 240-mg liver segments were hydrolyzed in $\mathrm{HCl}$, to which $40 \mathrm{mg}$ of Norit/Dowex mixture was added for $21 \mathrm{~h}$ at $110^{\circ} \mathrm{C}$. Following centrifugation and filtration, the $\mathrm{pH}$ was adjusted. Aliquots measuring $50 \mu \mathrm{l}$ were mixed with isopropanol and chloramine T/citrate buffer, $\mathrm{pH}$ 6. Ehrlich's reagent (Sigma, Saint Louis, MO, USA) was then added and the mixture was incubated at $60^{\circ} \mathrm{C}$ for $25 \mathrm{~min}$. Absorbance was recorded at $570 \mathrm{~nm}$. Hydroxyproline levels were calculated based on standard curves of 4-hydroxy-1-proline and expressed as micrograms per gram tissue.

\section{HSC Clones}

CFSC-8B and CFSC-3H, HSC clones isolated from a cell line derived from a single cirrhotic Wistar male albino rat, were provided by a collaborator (M Rojkind). ${ }^{25,26}$ The cells were maintained in DMEM containing 10\% fetal calf serum, $1 \%$ penicillin/streptomycin/nystatin and 1\% NEAA (Biological Industries, Beit Haemek, Israel). The concentrations of HF used were deduced previously, ${ }^{27}$ and are comparable to the ones reported by Schuppan and co-workers. ${ }^{28}$

\section{Real-time PCR}

Total RNA was extracted from $30 \mathrm{mg}$ liver samples or from lysed HSCs using the Qiagen RNeasy kit (Qiagen, Venlo, the Netherlands). RNA $(1 \mu \mathrm{g})$ was reverse transcribed using appropriate buffer, dNTP mix (Promega, Madison, WI, USA), RNasin (Promega) and MML-V reverse transcriptase. The ensuing cDNA was analyzed by real-time quantitative PCR with an ABI Prism 7000 sequence detection system (Applied Biosystems, Weiterstadt, Germany). GAPDH mRNA was used to normalize the level of cDNA. The results were evaluated with iCycler iQ Real-Time PCR Detection System (Applied Biosystems). Primers and probes were developed using Primer Express 2.0 software (Applied Biosystems). Rat primer sequences were as follows: procollagen- $\alpha(\mathrm{I})-1$, forward: CATTGGGTTCTCGGACTATTGAA, reverse: CCATG GAGATGCCAGATGGT; $\alpha$-SMA, forward: CGAAGCGCA GAGCAAGAGA, reverse: CATGTCGTCCCAGTTGGTGAT; VEGF-164, forward: AGAAAGCCCATGAAGTGGTG, reverse: ATCCCAGGGCTTCATCATTG; and heparanase: PrimerDesign (PrimerDesign, Southampton, UK). Human primers and probes were VEGF-165, heparanase: Assay-On-Demand (Applied Biosystems).

\section{Hydrodynamics-Based Transfection}

Hydrodynamics-based liver cell transfection was performed as previously described. ${ }^{29}$ Briefly, Ringer's solution equivalent to $5.25 \%$ of the body weight of a rat, containing $100 \mu \mathrm{g}$ of plasmid DNA encoding VEGF-165, was injected through the 
tail vein in 5-7 s. Prior to injection, rats were anesthetized using Ketamin $\mathrm{HCl}(90 \mathrm{mg} / \mathrm{kg})$ and Acepromazin Maleate PB $(1 \mathrm{mg} / \mathrm{kg})$. Ringer's solution was injected under the same conditions as control.

\section{Statistical Analysis}

Data are presented as mean \pm s.d. Statistical significance was determined by two-tailed Student's $t$-test and $P<0.05$ was considered significant.

\section{RESULTS}

\section{Liver Injury is Associated with Corresponding Heparanase and VEGF Expression}

TAA administration, twice weekly, resulted in severe liver injury characterized by a micronodular pattern with established fibrous septa linking portal tracts with each other and portal tracts to central veins (data not shown). Following cessation of treatment, a gradual decrease in hydroxyproline content, as well as improved histology, was evident at the two time points tested, 6 and 10 weeks into spontaneous recovery (Figure 1). Pro-collagen- $\alpha(\mathrm{I})-1$ mRNA level was elevated corresponding with the above, reaching its highest at peak fibrosis and returning to near-normal values 10 weeks into recovery (Figure 2). $\alpha$-SMA mRNA level, a bona fide marker of activation of HSC, increased significantly during development of fibrosis, reaching its highest 6 weeks following cessation of TAA injections, thereby receding to near-normal values at 10 weeks post TAA treatment cessation (Figure 2). Both heparanase and VEGF mRNA levels decreased at the early stage of liver injury, 1 and 3 weeks into TAA treatment, subsequently rising to their highest level at peak fibrosis. The levels of both gradually returned to normal values following removal of the fibrogenic agent.

\section{Correlated Expression of Heparanase and VEGF in the Rat Model of Fibrosis and HSCs}

A correlation between expression of heparanase and VEGF has previously been documented in cell lines stably transfected with heparanase. Such cells expressed elevated levels of VEGF. $^{30}$ In the present study, we tested whether overexpression of human VEGF in vivo affects the expression of heparanase as well. For this purpose, we used the hydrodynamics-based transfection (HBT) technology that has proven effective in our laboratory in both mouse and rat models using various genes. In a previous study, we showed that fibrosis causes a decline in the expression of hydrodynamically transfected genes and that HF, an inhibitor of collagen type I, partially reverses this effect. ${ }^{29}$ When transfected in the absence of HF, an approximately 100-fold increase in VEGF mRNA levels was evident compared with those in non-transfected, normal animals. The addition of HF for 10 weeks was associated with an additional significant increase of VEGF mRNA expression (Figure 3). In fibrotic rat liver recovering spontaneously from TAA, heparanase mRNA levels significantly decreased as compared with peak fibrosis.
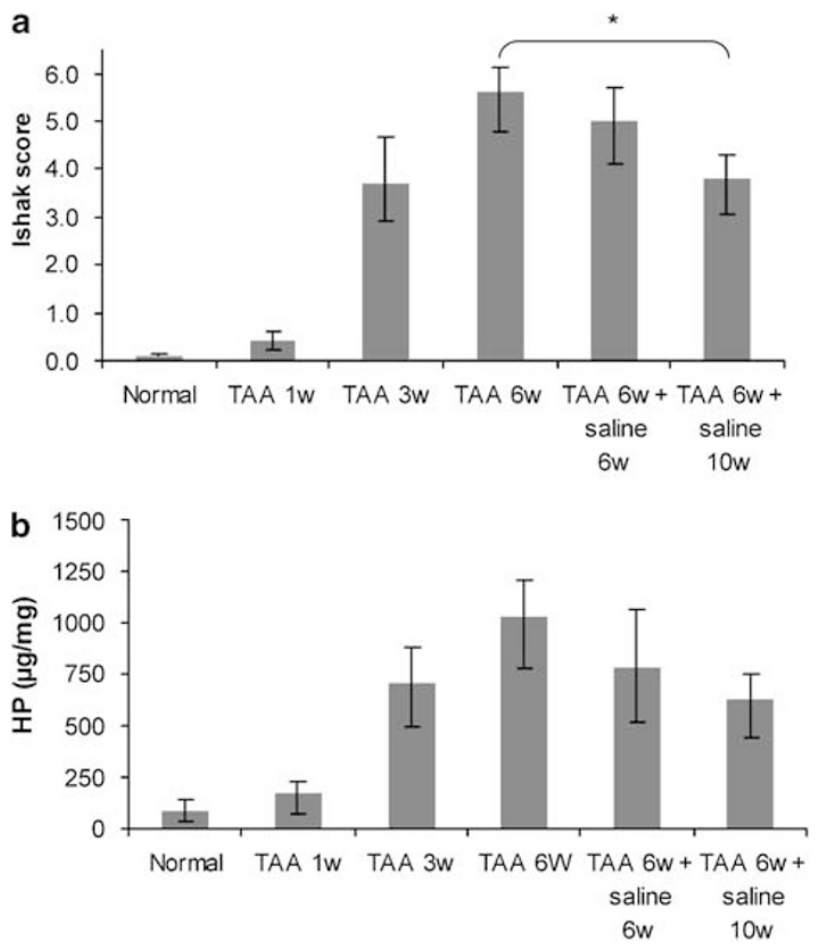

Figure 1 Histological (a) and chemical (hydroxyproline) (b) indices of liver injury in TAA-challenged rats. Paraformaldehyde-fixed liver sections were dehydrated in graded ethanol concentrations and stained with Sirius Red. The grade of fibrosis was determined based on the Ishak scoring system. Liver hydroxyproline content was measured following hydrolysis in $\mathrm{HCl}$ and use of chloramine T/citrate buffer and Ehrlich's reagent. Optical density was recorded at $570 \mathrm{~nm}$. 4-Hydroxy-1-proline was used to generate a standard curve $\left(n=5,{ }^{\star} P<0.05\right)$.

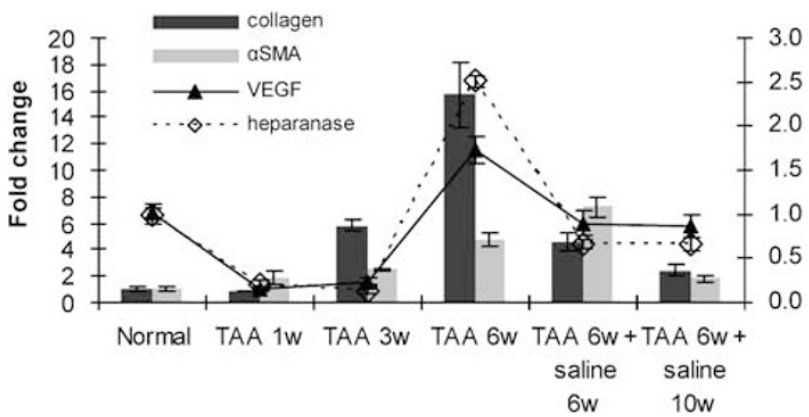

Figure 2 Collagen, $\alpha$-SMA, heparanase and VEGF-164 mRNA levels in TAAchallenged rats for varying time periods and also following elimination of the fibrogenic reagent using real-time PCR. mRNA levels were determined and standardized $v$ s levels of GAPDH $(n=5)$.

Transfection of liver cells with the human VEGF-165 using the HBT approach was nevertheless accompanied by a fourfold increase in heparanase mRNA levels. HF treatment, which allows better expression of the transfected gene, resulted in a further significant increase of heparanase mRNA transcripts. Of note is the fact that HF treatment by itself had a twofold stimulatory effect on heparanase expression (Figure 4). 


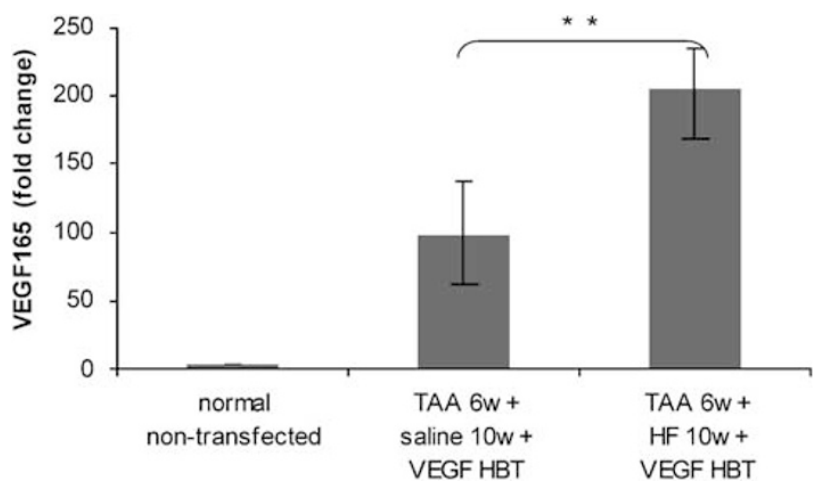

Figure 3 VEGF-165 mRNA levels in TAA-challenged rats subjected to HBT using a plasmid encoding human VEGF-165. Rats were treated with either $\mathrm{HF}$ or saline prior to HBT injection. Rats were administered a Ringer's solution equivalent to $5.25 \%$ of their body weight, containing $100 \mu \mathrm{g}$ of plasmid, in $5-7 \mathrm{~s}$. The levels of mRNA were determined $24 \mathrm{~h}$ post HBT by real-time PCR and standardized vs levels of GAPDH $\left(n=5,{ }^{*} P<0.01\right)$.

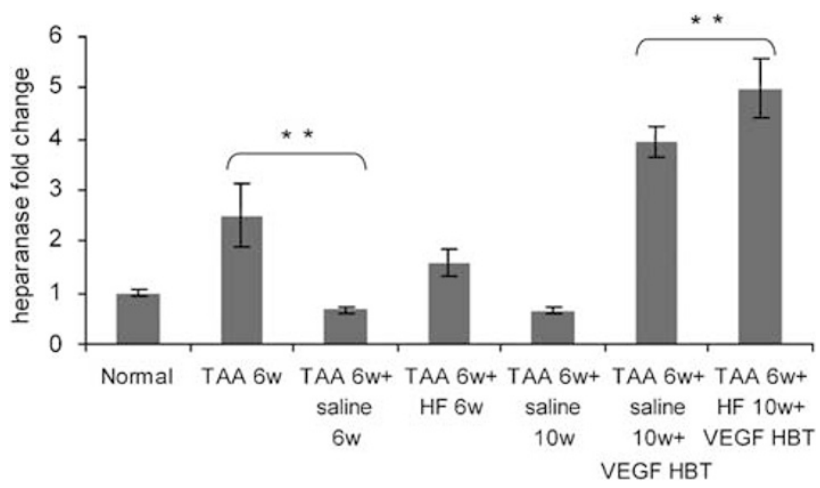

Figure 4 Heparanase mRNA levels in TAA-challenged rats allowed to either spontaneously recover (saline-injected) or treated with HF. Both groups were subjected to HBT using a plasmid encoding VEGF-165 gene. The levels of mRNA were determined $24 \mathrm{~h}$ post HBT by real-time PCR and standardized vs levels of GAPDH $\left(n=5,{ }^{*} P<0.01\right)$.

\section{Fibrotic Rat Livers Overexpressing VEGF-165 Demonstrate Improved Regeneration}

Liver regeneration is an orchestrated event controlled by various factors, including angiogenic mediators. ${ }^{31,32}$ Given the diverse properties of heparanase in various biological processes, we next compared the capacity of the various groups of rats to respond to 70\% PHx. Following 6 weeks of TAA treatment, rats undergoing $70 \% \mathrm{PHx}$ demonstrated poor capacity to regenerate, gaining only $18 \%$ restituted liver mass 5 days post hepatectomy. Healthy animals, in contrast, gained 55\% restituted liver mass. Notably, the combination of HF treatment during the 10 weeks of recovery followed by HBT of VEGF significantly increased restituted liver mass at 5 days post hepatectomy to close to $40 \%$. Neither spontaneous recovery for 10 weeks followed by HBT of either VEGF-165 or Ringer, nor HF treatment during the 10 weeks of recovery followed by HBT of Ringer, significantly augmented liver regeneration (Figure 5).

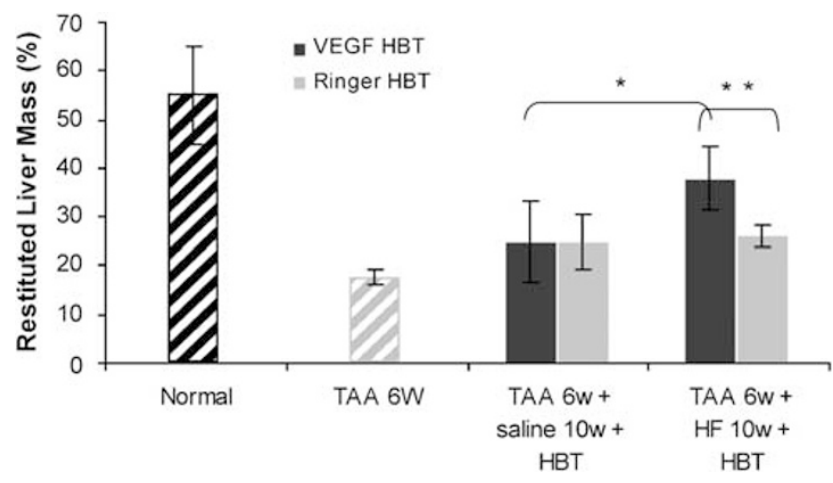

Figure 5 Liver regenerations following $70 \% \mathrm{PHx}$ in rats were treated with TAA for 6 weeks and subsequently with HF or saline for a further 10 weeks at which time point they were subjected to HBT using a plasmid $(100 \mu \mathrm{g})$ encoding the VEGF-165 gene or Ringer's solution. PHx was performed by removing the median and left lateral lobes. Liver regeneration was evaluated by calculating percentage restituted liver mass 5 days post surgery $\left(n=5,{ }^{* *} P<0.01,{ }^{\star} P<0.05\right)$.

\section{HF Upregulates the Expression of Heparanase and VEGF mRNA in HSCs Grown In Vitro}

Given the stimulatory effect of HF on the expression of heparanase in vivo, we next determined whether HF exerts a similar effect in vitro in CFSC-8B and CFSC-3H HSC cell lines derived from cirrhotic rat. The cells express basic levels of collagen type I, heparanase and VEGF mRNA. When grown in the presence of increasing concentrations of HF, the expression levels of heparanase and VEGF-165 mRNA gradually elevated, peaking at $50 \mathrm{nM} \mathrm{HF}$. The level of collagen type I mRNA was inhibited as expected, confirming previous reports ${ }^{17}$ (Figure 6).

\section{DISCUSSION}

Liver fibrosis is characterized by increased deposition of ECM, of which collagen and proteoglycans are major components. In the advanced form of the disease, cirrhosis, dense bands of collagen linking vascular structures and surrounding islands of regenerating parenchymal cells are evident. Several studies of either human patients or animal models have shown that recovery and remodeling of fibrotic liver is possible. ${ }^{5,33,34}$ We have used TAA to induce liver fibrosis in rats. Based on histology and hydroxyproline concentration, 6 weeks of TAA administration twice weekly inflicted liver damage graded as advanced fibrosis according to the Ishak scoring system. Six and 10 weeks of spontaneous recovery (saline injections) were, as expected, associated with gradual reduction in both hydroxyproline content and histology scoring. These results were further supported by a corresponding decline in collagen mRNA. Heparanase follow-up during development of fibrosis supports our assumption that the enzyme is involved in the massive changes associated with this type of liver injury. Of special note is the fact that VEGF and heparanase mRNA levels demonstrated a similar pattern, dropping to lower levels at weeks 1 and 3 of TAA treatment, 


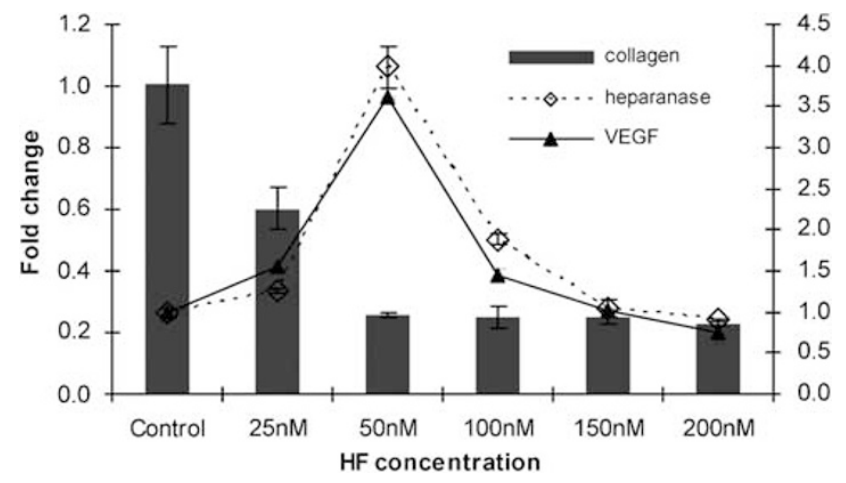

Figure 6 Collagen, heparanase and VEGF-164 mRNA levels in CFSC-8B HSC lines treated with increasing concentrations of HF. Cells were incubated with increasing concentrations of $\mathrm{HF}$ for $48 \mathrm{~h}$ and RNA was extracted. mRNA levels were determined by real-time PCR and standardized vs levels of GAPDH $(n=5)$.

followed by an increase to their highest level at peak fibrosis. Six and 10 weeks following TAA treatment cessation were associated with a reduction in mRNA levels of the two genes to near-normal levels (Figure 2). Heparanase activity is traditionally associated with modulating of the molecular structure of ECM and subendothelial basement membrane, resulting in release of various factors, thereby affecting normal and pathological processes. ${ }^{9,35}$ Heparanase has also been shown to exert non-enzymatic effects, including phosphorylation of a number of factors. ${ }^{36,37}$ Its translocation to the nucleus in various cell types may be an additional, potential, mechanism affecting gene transcription. ${ }^{38}$ We have documented a biphasic gain of heparanase expression during liver regeneration following $70 \% \mathrm{PHx}^{15}$

In a previous study, we used HBT to introduce the human VEGF-165 gene into liver cells. This procedure allowed VEGF expression for 17 days post administration and was significantly less effective in rats with TAA-induced liver fibrosis (Yeikilis et $a l^{29}$ and unpublished data). HF, which was demonstrated by us and others to reduce collagen type I mRNA level and synthesis, and thus decrease collagen deposition in TAA-challenged rat livers, was found to improve the efficiency of HBT. In the present study, 10 weeks of HF treatment prior to HBT resulted in significantly elevated expression of HBT-injected VEGF-165 mRNA compared with that in saline-treated rats (Figure 3). Heparanase mRNA levels in the above groups of animals corresponded to VEGF levels (Figure 4). Of special interest is the fact that HF treatment for 6 weeks post cessation of TAA induced a twofold increase in heparanase mRNA transcripts as compared with that in saline-treated rats (Figure 4). When combined with HBT of human VEGF-165, heparanase mRNA levels further increased fivefold compared with normal levels. HBT transfection of liver cells with VEGF-165 in the absence of HF was associated with a fourfold increase in heparanase mRNA. It follows, therefore, that both overexpression of VEGF-165 and HF treatment stimulate heparanase expression in vivo. Comparable results were obtained in vitro following transfection of CFSC- $8 \mathrm{~B}$ and CFSC- $3 \mathrm{H}$ with a three-plasmid lentiviral system containing pTK208 encoding human VEGF-165, the $\triangle$ NRF packaging vector and VSVG envelope vector. Such cells exhibited a fivefold increase in heparanase mRNA levels as compared to non-transfected cells (data not shown).

To rule out the possibility that the increase in heparanase mRNA expression is the result of an improved transfection of liver cells following HBT due to reduced fibrotic load, we next tested the effect of HF on the expression of heparanase in two HSC lines. In both cell lines tested, CFSC-3H and CFSC-8B, a remarkable increase in heparanase mRNA levels was documented at low concentrations ( $50 \mathrm{nM})$ of HF. Concentrations higher than $50 \mathrm{nM}$ were inhibitory to heparanase expression, although did not affect cell proliferation (data not shown). A similar pattern was obtained with VEGF, reminiscent of the expression of the two genes in rats developing fibrosis (Figures 2 and 6). In both models, the level of collagen type I mRNA was reduced by HF, confirming other reports. $^{39}$

A stimulatory effect of heparanase on VEGF expression was previously documented in vitro. ${ }^{30}$ In various cell lines, such as human embryonic kidney, breast carcinoma and rat glioma, overexpression of heparanase resulted in a 3- to 6 -fold increase in VEGF protein and mRNA levels. This increase was correlated with elevation of p38 phosphorylation. Moreover, partially blocking heparanase mRNA expression by applying a specific siRNA was accompanied by decrease in VEGF and $\mathrm{p} 38$ phosphorylation. ${ }^{30}$ These results suggest that expression of VEGF is regulated by heparanase. In that respect, the present study adds another component to the regulation interplay of heparanase and VEGF, namely, overexpression of VEGF in fibrotic rat liver leads to increased expression of heparanase, and HF stimulates expression of both heparanase and VEGF in HSCs.

Liver regeneration involves an orchestrated expression of a wide range of factors each of which is expressed at a defined time frame. These factors affect many processes, including cell migration, ECM remodeling and angiogenesis. Among the growth- and angiogenesis-promoting factors released by heparanase from the ECM are VEGF, bFGF, HGF and HBEGF, which have all been associated with liver regeneration and remodeling. ${ }^{40}$ We have previously demonstrated a stimulatory effect of exogenous VEGF-165 and VEGF-121 on liver regeneration following $\mathrm{PHx}^{41,42}$ We have also documented a gain of heparanase expression following $70 \%$ PHx. ${ }^{15} \mathrm{HF}$ treatment, which was used in our animal model to reduce collagen deposition, ${ }^{20}$ elevated VEGF-165 expression and also stimulated heparanase expression. These rats exhibited a better capacity to regenerate after $70 \% \mathrm{PHx}$ compared with non-VEGF-165-transfected animals, pointing to the contribution of both genes to the capacity of the injured liver to regenerate.

Apoptosis of activated HSCs during recovery leads to reduction in both hepatic collagen production and TIMP 
expression, thereby resulting in collagenolysis. ${ }^{5,6} \mathrm{HF}$ has been shown to have a pronounced stimulatory effect on matrix metalloproteinase-3 (MMP-3) and MMP-13, and an inhibitory effect on MMP-2, thereby promoting the collagenolytic activity. ${ }^{28} \mathrm{HF}$ has been shown by us and others to reduce collagen and $\alpha$-SMA in fibrotic rat liver, further assisting liver remodeling. Interestingly, the decrease in collagen content by itself may affect survival of HSCs. Indeed, recent studies showed that intact collagen type I protects HSCs from apoptosis following cessation of liver injury, ${ }^{43}$ further supporting the potential of $\mathrm{HF}$ as a therapeutic drug.

TGF- $\beta$ is considered a major pro-fibrogenic cytokine. As such many have tested whether the inhibitory effect of HF on the synthesis of collagen type I is mediated through the TGF- $\beta$-signaling pathway. Bona and co-workers ${ }^{44}$ have demonstrated that HF inhibits TGF- $\beta$-induced upregulation of collagen by interfering with the phosphorylation and subsequent activation of Smad-3. In a transcriptional profiling survey of rats treated with TAA and HF, we analyzed genes that are associated with TAA-induced fibrosis. In this study, we identified changes in $7 \%$ of liver genes; HF prevented expression of $41 \%$ of those genes. In fact, HF affects different genes at the early stages of the disease that are involved in alcohol, lipid, protein and phosphate metabolism, and in cell adhesion. In later stages, HF affects genes involved in cell development, proliferation, differentiation and apoptosis. ${ }^{18}$

We cannot exclude that a component of fibrogenesis in this model is contributed by portal-tract myofibroblasts. ${ }^{45}$ However, the primary site of TAA cytotoxicity and fibrogenesis is centrilobular. ${ }^{46,47}$ In a recent study using rat liver precision slices, TAA was shown to induce hepatocellular cytotoxicity in a periportal location as well. ${ }^{48}$ However, portal-tract fibrogenesis was not a prominent feature in this model, and was better induced following treatment with $\beta$-estradiol or epidermal growth factor (EGF). The finding of TAA inducing predominantly parenchymal fibrosis is in keeping with our previously published work ${ }^{49}$ in which immunohistochemistry demonstrated parenchymal deposition of fibrous tissue following TAA administration.

In summary, the improved capacity of rat liver regeneration exhibited by a combined therapy of HF and VEGF transfection may in fact be the result of the various effects exerted by HF. These include induction of heparanase and VEGF expression (this communication); stimulation of MMP-3 and MMP-13; downregulation of MMP-2 and influence on HSC proliferation; ${ }^{28}$ blockade of collagen type I synthesis (this communication and Granot et $a l^{16}$ ); and the ability to enhance regenerative response. ${ }^{20}$ Although it is known that HF inhibits TGF- $\beta$ expression, the main fibrogenic cytokine, the mechanism of action of HF is still to be elucidated. HF has been marketed for years for use in animals raised for human consumption. Its antifibrotic activity renders this compound particularly suitable for further evaluation in the treatment of liver fibrosis, where no other remedies have been found.

\section{ACKNOWLEDGEMENT}

We thank Dr Israel Vlodavsky for continuous support and fruitful discussion throughout this study. This work was supported by Israel Cancer Research Foundation 800005, Israel Science Foundation 1256/07, the A \& E Blum Medical Research Fund, and the RO1 AA10541 and AA09231 (MR) funds.

\section{DISCLOSURE/DUALITY OF INTEREST}

None of the authors has any duality of interest to declare.

1. Rojkind M, Giambrone MA, Biempica L. Collagen types in normal and cirrhotic liver. Gastroenterology 1979;76:710-719.

2. Guo J, Friedman SL. Hepatic fibrogenesis. Semin Liver Dis 2007;27: 413-426.

3. Iredale JP. Models of liver fibrosis: exploring the dynamic nature of inflammation and repair in a solid organ. J Clin Invest 2007;117: 539-548.

4. Murphy FR, Issa R, Zhou X, et al. Inhibition of apoptosis of activated hepatic stellate cells by tissue inhibitor of metalloproteinase- 1 is mediated via effects on matrix metalloproteinase inhibition: implications for reversibility of liver fibrosis. J Biol Chem 2002;277:11069-11076.

5. Iredale JP, Benyon RC, Pickering J, et al. Mechanisms of spontaneous resolution of rat liver fibrosis. Hepatic stellate cell apoptosis and reduced hepatic expression of metalloproteinase inhibitors. J Clin Invest 1998;102:538-549.

6. Issa R, Zhou X, Constandinou CM, et al. Spontaneous recovery from micronodular cirrhosis: evidence for incomplete resolution associated with matrix cross-linking. Gastroenterology 2004;126:1795-1808.

7. Kjellen L, Lindahl U. Proteoglycans: structures and interactions. Annu Rev Biochem 1991;60:443-475.

8. David G. Integral membrane heparan sulfate proteoglycans. FASEB J 1993;7:1023-1030

9. Vlodavsky I, Friedmann Y. Molecular properties and involvement of heparanase in cancer metastasis and angiogenesis. J Clin Invest 2001;108:341-347.

10. Parish CR, Freeman C, Hulett MD. Heparanase: a key enzyme involved in cell invasion. Biochim Biophys Acta 2001;1471:M99-M108.

11. Nakajima M, Irimura T, Nicolson GL. Heparanases and tumor metastasis. J Cell Biochem 1988;36:157-167.

12. Vlodavsky I, Friedmann $Y$, Elkin $M$, et al. Mammalian heparanase: gene cloning, expression and function in tumor progression and metastasis. Nat Med 1999;5:793-802.

13. Dempsey LA, Brunn GJ, Platt JL. Heparanase, a potential regulator of cell-matrix interactions. Trends Biochem Sci 2000;25:349-351.

14. Ilan N, Elkin M, Vlodavsky I. Regulation, function and clinical significance of heparanase in cancer metastasis and angiogenesis. Int J Biochem Cell Biol 2006;38:2018-2039.

15. Goldshmidt $\mathrm{O}$, Yeikilis $\mathrm{R}$, Mawasi $\mathrm{N}$, et al. Heparanase expression during normal liver development and following partial hepatectomy. J Pathol 2004;203:594-602.

16. Granot I, Halevy O, Hurwitz S, et al. Halofuginone: an inhibitor of collagen type I synthesis. Biochim Biophys Acta 1993;1156:107-112.

17. Pines $M$, Nagler A. Halofuginone: a novel antifibrotic therapy. Gen Pharmacol 1998;30:445-450.

18. Gnainsky Y, Kushnirsky Z, Bilu G, et al. Gene expression during chemically induced liver fibrosis: effect of halofuginone on TGF-beta signaling. Cell Tissue Res 2007;328:153-166.

19. Pines M. Targeting TGF-beta signaling to inhibit fibroblast activation as a therapy for fibrosis and cancer effect of halofuginone. Exp Opinion Drug Discov 2008;3:1-10.

20. Spira G, Mawasi N, Paizi M, et al. Halofuginone, a collagen type I inhibitor improves liver regeneration in cirrhotic rats. J Hepatol 2002;37:331-339.

21. Ishak K, Baptista A, Bianchi L, et al. Histological grading and staging of chronic hepatitis. J Hepatol 1995;22:696-699.

22. Lopez-De Leon A, Rojkind M. A simple micromethod for collagen and total protein determination in formalin-fixed paraffin-embedded sections. J Histochem Cytochem 1985;33:737-743.

23. Jamall IS, Finelli VN, Que Hee SS. A simple method to determine nanogram levels of 4-hydroxyproline in biological tissues. Anal Biochem 1981;112:70-75. 
24. Jonsson JR, Clouston AD, Ando $\mathrm{Y}$, et al. Angiotensin-converting enzyme inhibition attenuates the progression of rat hepatic fibrosis. Gastroenterology 2001;121:148-155.

25. Greenwel $P$, Schwartz $M$, Rosas $M$, et al. Characterization of fat-storing cell lines derived from normal and $\mathrm{CCl} 4$-cirrhotic livers. Differences in the production of interleukin-6. Lab Invest 1991;65:644-653.

26. Greenwel P, Rubin J, Schwartz $M$, et al. Liver fat-storing cell clones obtained from a CCl4-cirrhotic rat are heterogeneous with regard to proliferation, expression of extracellular matrix components, interleukin-6, and connexin 43. Lab Invest 1993;69:210-216.

27. Gnainsky $Y$, Spira G, Paizi $M$, et al. Halofuginone, an inhibitor of collagen synthesis by rat stellate cells, stimulates insulin-like growth factor binding protein-1 synthesis by hepatocytes. J Hepatol 2004:40:269-277.

28. Popov $Y$, Patsenker E, Bauer $M$, et al. Halofuginone induces matrix metalloproteinases in rat hepatic stellate cells via activation of p38 and NFkappaB. J Biol Chem 2006;281:15090-15098.

29. Yeikilis R, Gal S, Kopeiko N, et al. Hydrodynamics based transfection in normal and fibrotic rats. World J Gastroenterol 2006;12: 6149-6155.

30. Zetser A, Bashenko Y, Edovitsky $E$, et al. Heparanase induces vascular endothelial growth factor expression: correlation with p38 phosphorylation levels and Src activation. Cancer Res 2006;66: 1455-1463.

31. Michalopoulos GK, DeFrances MC. Liver regeneration. Science 1997;276:60-66.

32. Fausto N. Liver regeneration. J Hepatol 2000;32:19-31.

33. Li D, Friedman SL. Liver fibrogenesis and the role of hepatic stellate cells: new insights and prospects for therapy. J Gastroenterol Hepatol 1999;14:618-633.

34. Benyon RC, Iredale JP. Is liver fibrosis reversible? Gut 2000;46:443-446.

35. Elkin M, Ilan N, Ishai-Michaeli R, et al. Heparanase as mediator of angiogenesis: mode of action. FASEB J 2001;15:1661-1663.

36. Ben-Zaken O, Gingis-Velitski S, Vlodavsky I, et al. Heparanase induces Akt phosphorylation via a lipid raft receptor. Biochem Biophys Res Commun 2007;361:829-834.

37. Gingis-Velitski S, Zetser A, Flugelman MY, et al. Heparanase induces endothelial cell migration via protein kinase B/Akt activation. J Biol Chem 2004;279:23536-23541.
38. Nobuhisa $T$, Naomoto $\mathrm{Y}$, Okawa $\mathrm{T}$, et al. Translocation of heparanase into nucleus results in cell differentiation. Cancer Sci 2007;98:535-540.

39. Nagler A, Firman N, Feferman R, et al. Reduction in pulmonary fibrosis in vivo by halofuginone. Am J Respir Crit Care Med 1996;154:1082-1086.

40. Su Al, Guidotti LG, Pezacki JP, et al. Gene expression during the priming phase of liver regeneration after partial hepatectomy in mice. Proc Natl Acad Sci USA 2002;99:11181-11186.

41. Assy N, Spira G, Paizi M, et al. Effect of vascular endothelial growth factor on hepatic regenerative activity following partial hepatectomy in rats. J Hepatol 1999;30:911-915.

42. Kraizer Y, Mawasi N, Seagal J, et al. Vascular endothelial growth factor and angiopoietin in liver regeneration. Biochem Biophys Res Commun 2001;287:209-215.

43. Zhou X, Jamil A, Nash A, et al. Impaired proteolysis of collagen inhibits proliferation of hepatic stellate cells: implications for regulation of liver fibrosis. J Biol Chem 2006;281:39757-39765

44. McGaha TL, Phelps RG, Spiera $\mathrm{H}$, et al. Halofuginone, an inhibitor of type-I collagen synthesis and skin sclerosis, blocks transforming growth-factor-beta-mediated Smad3 activation in fibroblasts. J Invest Dermatol 2002;118:461-470.

45. Ramadori G, Saile B. Portal tract fibrogenesis in the liver. Lab Invest 2004;84:153-159.

46. Krull NB, Zimmermann T, Gressner AM. Spatial and temporal patterns of gene expression for the proteoglycans biglycan and decorin and for transforming growth factor-beta 1 revealed by in situ hybridization during experimentally induced liver fibrosis in the rat. Hepatology 1993; 18:581-589.

47. Novosyadlyy R, Dargel R, Scharf JG. Expression of insulin-like growth factor-I and insulin-like growth factor binding proteins during thioacetamide-induced liver cirrhosis in rats. Growth Horm IGF Res 2005; 15:313-323.

48. Guyot C, Combe C, Clouzeau-Girard H, Moronvalle-Halley V, Desmoulière A. Specific activation of the different fibrogenic cells in rat cultured liver slices mimicking in vivo situations. Virchows Arch 2007;450:503-512.

49. Bruck R, Genina $\mathrm{O}$, Aeed $\mathrm{H}$, et al. Halofuginone to prevent and treat thioacetamide-induced liver fibrosis in rats. Hepatology 2001;33: 379-386. 